\title{
Landau-fluid simulations of Alfvén-wave instabilities in a warm collisionless plasma
}

\author{
G. Bugnon, T. Passot, and P. L. Sulem \\ CNRS, Observatoire de la Côte d'Azur, B.P. 4229, 06304 Nice Cedex 4, France
}

Received: 6 October 2004 - Revised: 10 November 2004 - Accepted: 12 November 2004 - Published: 30 November 2004

Part of Special Issue "Advances in space environment turbulence"

\begin{abstract}
A dispersive Landau-fluid model is used to study the decay and modulational instabilities of circularlypolarized Alfvén waves in a collisionless plasma, as well as their nonlinear developments. Comparisons are presented with the drift-kinetic approximation for the dispersionless regime and with hybrid simulations in more general conditions. The effect of the nature of the instability on particle heating is discussed, together with the formation of coherent structures or the development of an inverse cascade.
\end{abstract}

\section{Introduction}

When a magnetized plasma is considered at scales smaller than the collision mean free path, the usual MHD description is no longer valid due to significant kinetic effects. Such a situation is usual in the solar wind or the magnetosphere, but also in the warm ionized phase of the interstellar medium where the fluctuation spectrum extends down to scales comparable to the ion inertial length and much below the ionneutral or Coulomb mean free paths (Scalo and Elmegreen, 2004). In such collisionless regimes, Landau damping provides an important dissipation mechanism for low-frequency waves through particle resonance. Furthermore, MHD waves are made dispersive under the effect of the Hall term and the electron pressure gradient in a generalized Ohm's law, as well as of finite Larmor radius (FLR) corrections in the pressure tensor. Fully kinetic descriptions, based on the VlasovMaxwell system or even on the reduced gyrokinetic equations, remain however much beyond the capabilities of the present day computers in situations involving a broad range of scales, as in turbulent regimes. It is thus important to develop a description retaining the main kinetic effects within fluid models of large-scale plasma dynamics.

It turns out that in a weakly nonlinear regime, and for scales large relatively to the ion Larmor radius, an efficient

Correspondence to: P. L. Sulem

(sulem@obs-nice.fr) description of the plasma dynamics is provided by Landaufluid models introduced by Snyder et al. (1997). This approach extends the usual MHD by including dynamical equations for the parallel and perpendicular pressures of each particles species, together with a closure approximation that expresses the heat fluxes (or possibly the fourth order moments) in terms of temperature and magnetic perturbations, in a way consistent with the linear kinetic theory in the case of a proton-electron plasma with bi-Maxwellian equilibrium distribution functions. This model was extended in Passot and Sulem (2003b, 2004b) by including dispersive effects, required to describe the formation of solitonic structures currently observed in the solar wind and the magnetosphere. This dispersive Landau fluid was demonstrated to accurately reproduce the dispersion relations and Landau damping rates of long-wavelength magnetosonic and Alfvén waves for any $\beta$ larger than the electron to proton mass ratio $m_{e} / m_{p}$ and any propagation angle $\alpha$, including kinetic Alfvén waves with a transverse wavenumber not exceeding the inverse proton inertial length (Passot and Sulem, 2004a). This model also reproduces the weakly nonlinear dynamics of these waves. Numerical simulations of oblique fast magnetosonic waves in a slab geometry shows in particular the onset of a coupling with Alfvén waves that results in the quenching of Landau dissipation after a time that is shorter when the wave amplitude is larger (Bugnon et al., 2004). An excellent agreement is also obtained for the growth rate of long-wavelength mirror modes. Furthermore, the multidimensional kinetic derivative nonlinear Schrödinger equation for parallel-propagating long Alfvén waves, derived from Vlasov-Maxwell equations by means of a reductive perturbative expansion (Passot and Sulem, 2003a), is reproduced when this expansion is performed on the dispersive Landaufluid model, up to the replacement of the plasma response function by its two or four pole Padé approximants.

The aim of the present paper is to use the dispersive Landau-fluid model described in Sect. 2 (and in a more general setting in the Appendix) to address the instability of right or left-hand circularly polarized Alfvén waves propagating 
along a uniform ambient magnetic field in a warm collisionless plasma. Comparisons are in particular made with predictions of the drift-kinetic approximation for the dispersionless limit (Inhester, 1990) (Sect. 3) and, when dispersion is relevant, with hybrid simulations where electrons are treated as an isothermal fluid (Vasquez, 1995) (Sect. 4). The nonlinear dynamics initiated by decay and modulational instabilities is also analyzed. The main conclusions are summarized in Sect. 5.

\section{A dispersive Landau-fluid model}

The dispersive Landau-fluid model derived and validated by comparison with the kinetic theory in Passot and Sulem (2004b) is briefly described in the Appendix. We are here concerned with parallel Alfvén waves whose dynamics is accurately described by a simplified version of this model (Passot and Sulem, 2003b). This simplified model mainly consists in retaining leading order FLR corrections to the pressure and neglecting the contributions of non-gyrotropic heat flux components. Since this model also describes oblique magnetosonic waves (except for quasi-transverse propagation for which vector heat fluxes are to be considered; Mikkhailovskiǔ and Smolyakov, 1985), it is convenient to consider an ambient magnetic field making an arbitrary angle $\alpha$ with the direction of propagation (taken as $x$ ). Measuring the density $\rho$ in units of the equilibrium value $\rho^{(0)}$, the magnetic field $b=\left(b_{x}=B_{0} \cos \alpha, b_{y}, b_{z}\right)$ in units of the ambient field $B_{0}$, the plasma velocity $\left(u_{x}, u_{y}, u_{z}\right)$ in units of a velocity $u_{0}$ (later taken as the Alfvén velocity $v_{A}=B_{0} / \sqrt{4 \pi \rho_{0}}$ ), the parallel and perpendicular pressures $p_{\| r}$ and $p_{\perp r}$ of each species $r$ in units of the equilibrium parallel proton pressure $p_{0}=p_{\| p}^{(0)}$ and the corresponding heat fluxes $q_{\| r}$ and $q_{\perp r}$ in units of $u_{0} p_{0}$, the dynamic equations in a slab geometry read

$$
\partial_{t} \rho+\partial_{x}\left(\rho u_{x}\right)=0
$$

$\partial_{t} u_{x}+u_{x} \partial_{x} u_{x}+\frac{\beta_{\| p}}{2 M_{a}^{2}} \frac{1}{\rho} \partial_{x}\left(p_{x x}+\pi_{x x}\right)+\frac{1}{M_{a}^{2}} \frac{1}{\rho} \partial_{x} \frac{|b|^{2}}{2}=0$

$\partial_{t} u_{y}+u_{x} \partial_{x} u_{y}+\frac{\beta_{\| p}}{2 M_{a}^{2}} \frac{1}{\rho} \partial_{x}\left(p_{x y}+\pi_{x y}\right)-\frac{b_{x}}{M_{a}^{2}} \frac{1}{\rho} \partial_{x} b_{y}=0$

$\partial_{t} u_{z}+u_{x} \partial_{x} u_{z}+\frac{\beta_{\| p}}{2 M_{a}^{2}} \frac{1}{\rho} \partial_{x}\left(p_{x z}+\pi_{x z}\right)-\frac{b_{x}}{M_{a}^{2}} \frac{1}{\rho} \partial_{x} b_{z}=0$

$\partial_{t} b_{y}=\partial_{x}\left(b_{x} u_{y}-u_{x} b_{y}+\frac{R_{p} b_{x}}{M_{a}} \frac{\partial_{x} b_{z}}{\rho}-\frac{\beta_{\| p}}{2} \frac{R_{p}}{M_{a}} \frac{1}{\rho} \partial_{x} p_{e, x z}\right)$

$\partial_{t} b_{z}=\partial_{x}\left(b_{x} u_{z}-u_{x} b_{z}-\frac{R_{p} b_{x}}{M_{a}} \frac{\partial_{x} b_{y}}{\rho}+\frac{\beta_{\| p}}{2} \frac{R_{p}}{M_{a}} \frac{1}{\rho} \partial_{x} p_{e, x y}\right)$

$$
\begin{aligned}
\partial_{t} p_{\| r} & +\partial_{x}\left(u_{x} p_{\| r}\right) \\
& +2 \frac{p_{\| r}}{|b|^{2}}\left(b_{x}^{2} \partial_{x} u_{x}+b_{x} b_{y} \partial_{x} u_{y}+b_{x} b_{z} \partial_{x} u_{z}\right) \\
& +\partial_{x}\left(\frac{b_{x}}{|b|} q_{\| r}\right)-2 q_{\perp r} \partial_{x} \frac{b_{x}}{|b|}=0
\end{aligned}
$$

$$
\begin{aligned}
& \partial_{t} p_{\perp r}+\partial_{x}\left(u_{x} p_{\perp r}\right)+p_{\perp r} \partial_{x} u_{x} \\
&-\frac{p_{\perp r}}{|b|^{2}}\left(b_{x}^{2} \partial_{x} u_{x}+b_{x} b_{y} \partial_{x} u_{y}+b_{x} b_{z} \partial_{x} u_{z}\right) \\
&+\partial_{x}\left(\frac{b_{x}}{|b|} q_{\perp r}\right)+q_{\perp r} \partial_{x} \frac{b_{x}}{|b|}=0 \\
&\left(\partial_{t}+u_{x} \partial_{x}+\frac{1}{\left(\frac{u_{0}}{v_{t h, r}}\right)} \frac{\cos \alpha}{\sqrt{\frac{8}{\pi}}\left(1-\frac{3 \pi}{8}\right)} \mathcal{H} \partial_{x}\right) q_{\| r} \\
&=\frac{\cos \alpha}{\left(\frac{u_{0}^{2}}{v_{t h, r}^{2}}\right)\left(1-\frac{3 \pi}{8}\right)} \partial_{x}\left(\frac{p_{\| r}}{\rho}\right) \\
&\left(\partial_{t}+u_{x} \partial_{x}-\frac{1}{\left(\frac{u_{0}}{v_{t h, r}}\right)} \cos \alpha \sqrt{\frac{\pi}{2}} \mathcal{H} \partial_{x}\right) q_{\perp r} \\
&=-\frac{\cos \alpha}{\left(\frac{u_{0}^{2}}{v_{t h, r}^{2}}\right)} \partial_{x}\left[\frac{p_{\perp r}}{\rho}+\left(\frac{p_{\perp r}^{(0)}}{p_{\| r}^{(0)}}-1\right) \frac{p_{\perp r}^{(0)}}{p_{0}}|b|\right],
\end{aligned}
$$

where the gyrotropic components of the pressure tensor are given by $p_{i j}=\sum_{r} p_{r, i j}$ with

$$
\begin{aligned}
& p_{r, x x}=p_{\perp r}+\left(p_{\| r}-p_{\perp r}\right) \frac{b_{x}^{2}}{|b|^{2}} \\
& p_{r, x y}=\left(p_{\| r}-p_{\perp r}\right) \frac{b_{x} b_{y}}{|b|^{2}} \\
& p_{r, x z}=\left(p_{\| r}-p_{\perp r}\right) \frac{b_{x} b_{z}}{|b|^{2}} .
\end{aligned}
$$

In the equations for the parallel and transverse heat fluxes, $\mathcal{H}$ denotes the Hilbert transform relatively to the $x$ coordinate, $v_{t h, r}=\sqrt{T_{\| r}^{(0)} / m_{r}}$ is the thermal velocity of the particles of species $r, M_{a}=u_{0} / v_{A}$ is the Alfvénic Mach number (here taken equal to 1 ) and $\beta_{\| p}=8 \pi p_{0} / B_{0}^{2}$. This parameter is related to the usual $\beta=8 \pi P_{0} / B_{0}^{2}$ by $\beta=\beta_{\| p}\left(1+T_{e}^{(0)} / T_{p}^{(0)}\right)$. Neglecting contributions involving the electron to proton mass ratio, one gets $\frac{u_{0}^{2}}{v_{t h, p}^{2}}=\frac{2 M_{a}^{2}}{\beta_{\| p}}$ and $\frac{u_{0}^{2}}{v_{t h, e}^{2}}=2 \frac{m_{e}}{m_{p}} \frac{p_{\| p}^{(0)}}{p_{\| e}^{(0)}} \frac{M_{a}^{2}}{\beta_{\| p}}$. Furthermore $R_{p}=\frac{v_{A}}{\Omega_{p} L_{0}}$ (where $\Omega_{p}=\frac{e B_{0}}{m_{p} c}$ is the proton $\mathrm{gy}$ rofrequency) measures the ratio of the proton inertial length to the reference length scale $L_{0}$. As a consequence, time is measured in units of $1 /\left(R_{p} \Omega_{p}\right)$. The non-gyrotropic (FLR) corrections in the pressure tensor are restricted to their leading order, in the form

$$
\begin{aligned}
\pi_{x x} & =M_{a} R_{p} \sin \alpha\left(2 \cos ^{2} \alpha\left(p_{\perp p}-2 p_{\| p}\right)-\frac{1}{2} \sin ^{2} \alpha p_{\perp p}\right) \partial_{x} u_{y} \\
\pi_{x y} & =M_{a} R_{p} \cos \alpha\left(\left(p_{\perp p}-2 p_{\| p} \cos ^{2} \alpha\right) \partial_{x} u_{z}+2 p_{\| p} \cos \alpha \sin \alpha \partial_{x} u_{x}\right) \\
& +M_{a} R_{p} \sin ^{2} \alpha p_{\perp p}\left(\sin \alpha \partial_{x} u_{x}-\cos \alpha \partial_{x} u_{z}\right)
\end{aligned}
$$



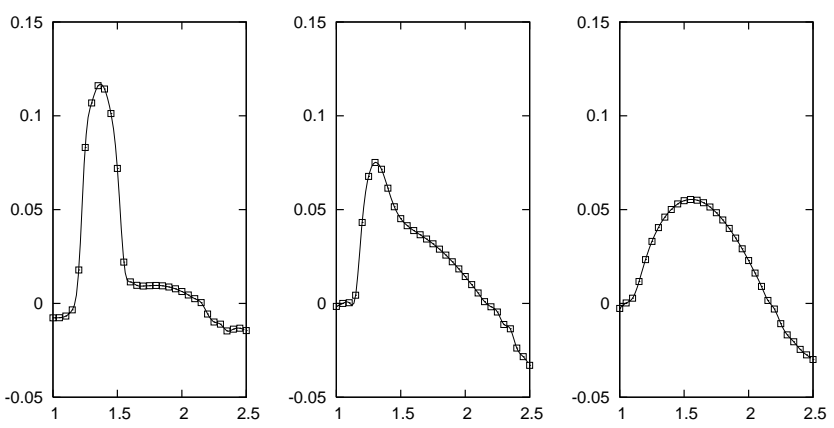

Fig. 1. Growth rates of the density modes (whose wavenumber is normalized by the pump wavenumber) resulting from the decay instability of a non dispersive Alfvén wave of amplitude $b_{0}=0.447$ propagating in a plasma with $\beta=0.6$ and isotropic equilibrium temperatures of the electrons and ions in a ratio $T_{e}^{(0)} / T_{p}^{(0)}=33$ (left), $T_{e}^{(0)} / T_{p}^{(0)}=5$ (middle) and $T_{e}^{(0)} / T_{p}^{(0)}=1$ (right).

$\pi_{x z}=M_{a} R_{p} \cos \alpha\left(\left(\sin ^{2} \alpha-\cos ^{2} \alpha\right)\left(p_{\perp p}-2 p_{\| p}\right)+\sin ^{2} \alpha p_{\perp p}\right) \partial_{x} u_{y}$.

This system of equations was recently used to analyze the dynamics of oblique magnetosonic waves (Bugnon et al., 2004). Here, we concentrate on parallel Alfvén waves and thus take $\alpha=0$ in the above system.

\section{The numerical set-up}

It is easily seen that parallel-propagating circularlypolarized monochromatic Alfvén waves characterized by the condition $\rho=1, b_{x}=1$, uniform and time-independent pressures, together with $b_{ \pm} \equiv b_{y} \pm i b_{z}=b_{0} e^{i(k x-\omega t)}$ and $u_{ \pm} \equiv u_{y} \pm i u_{z}=u_{0} e^{i(k x-\omega t)}$, are exact solutions of the dispersive Landau-fluid model. Here, the - and + signs refer to right and left-hand polarizations, respectively. They obey

$\left(\omega \pm k^{2} R_{p} \alpha_{e}\right) b_{ \pm}+k u_{ \pm}=0$

$\left(\omega \mp \frac{\beta}{2} R_{p}\left(p_{\perp}^{(0)}-2 p_{\|}^{(0)}\right) k^{2}\right) u_{ \pm}+\alpha k b_{ \pm}=0$,

with $\alpha_{e}=1+\frac{\beta\left(p_{\perp e}^{(0)}-p_{\| e}^{(0)}\right)}{2\left(1+\left|b_{0}\right|^{2}\right)}$ and $\alpha=1+\frac{\beta\left(p_{\perp}^{(0)}-p_{\|}^{(0)}\right)}{2\left(1+\left|b_{0}\right|^{2}\right)}$.

Note that these waves are solutions of the Landau-fluid model even for isotropic pressures (or temperatures), while in a Vlasov-Maxwell description, pressure anisotropy is required (Abraham-Shrauner and Feldman, 1977). In the hybrid simulations presented by Vasquez (1995), the initial condition is not an exact solution and an adjustment phase involving pressure anisotropies is visible. This constraint is however relaxed in the drift-kinetic approximation (Inhester, 1990) that, like the Landau fluids, deals with the longwavelength limit.

The above Landau-fluid equations are integrated using a pseudo-spectral method in a periodic domain and a second order scheme for the time stepping. Resolutions of

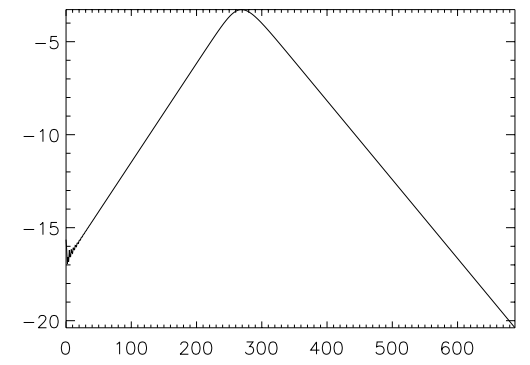

Fig. 2. Time evolution of the amplitude of the density mode $k=1.5$ in lin-log scales, for the conditions of Fig. 1 (right).

128 or 256 collocation points are used. The initial conditions are taken as a right or left-hand polarized wave. Except when comparing with previously published growth rate curves where it is necessary to add a perturbation at a selected wavenumber, we rely on the numerical noise to destabilize the solution. It turns out that when aliasing errors are not eliminated, the code rapidly becomes unstable for initial wave amplitudes exceeding a few tenths. Since the nonlinearities of the model are not polynomials, aliasing cannot be totally eliminated. Nevertheless, a partial desaliasing where all the modes with wavenumbers larger than one half the largest wavenumber permitted by the resolution are put to zero, appears sufficient to stabilize the simulation and to ensure accuracy (as checked by increasing space and time resolution). Furthermore, the dispersive effects (treated by a semi-implicit scheme because of the involved nonlinearities) requires a time step significantly smaller than suggested by the usual stability criteria.

\section{The dispersionless limit}

The decay instability of parallel Alfvén waves propagating in a warm collisionless plasma was analyzed by Inhester (1990) in the case where the ratio of the Larmor radius to the pump wavelength is asymptotically small, making dispersion immaterial, as for example in the solar wind (except near the Earth). This situation permits the use of the so-called driftkinetic approximation, obtained as the leading-order result of the averaging over the particle gyromotion, that leads to dynamical equations for the distribution functions of the ion and electron guiding centers (see e.g. Grad, 1961; Kulsrud, 1983, Sulem, 2004 ${ }^{1}$, and references therein). We here only consider the case of bi-Maxwellian equilibrium distribution functions, for consistency with the assumptions used for the Landau-fluid model.

Decay instability produces a forward propagating acoustic wave and a backward Alfvén wave with a wavenumber smaller than that of the pump. In Fig. 1 of Inhester (1990), the growth rate (normalized by the pump frequency)

\footnotetext{
${ }^{1}$ Sulem, P. L.: Introduction to the guiding center theory, in Proc. Course and Workshop on Kinetic Theory (Toronto, March 2004), Fields Communications Series, to appear, 2004.
} 


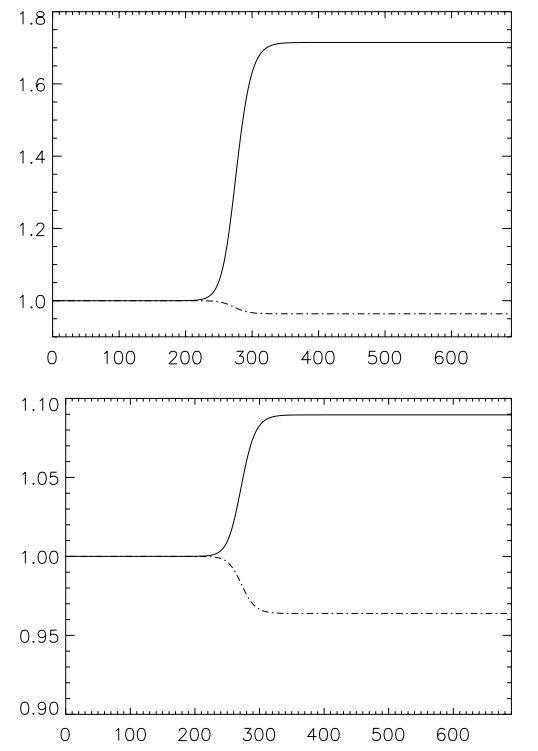

Fig. 3. Time evolution of parallel (solid lines) and transverse (dashed-dotted lines) mean temperatures of the ions (top) and the electrons (bottom) in the run of Fig. 1 (right).

of the acoustic mode is displayed versus the perturbation wavenumber, for a circularly polarized pump wave of amplitude $\sqrt{0.2} \approx 0.447$ and various values of the ratio $p_{p}^{(0)} / p_{e}^{(0)}$ of the isotropic equilibrium proton to electron pressures (or temperatures), the total thermal pressure $P_{0}=p_{p}^{(0)}+p_{e}^{(0)}$ being kept constant and prescribed by the value of the parameter $\beta=8 \pi P_{0} / B_{0}^{2}$. We show in Fig. 1 that Landau-fluid simulations reproduce reasonably well the instability growth rates displayed in Inhester (1990) except that we had to use $\beta_{\| p}=0.3$, which corresponds to $\beta=0.6$, one half the value indicated in Inhester's paper. The same discrepancy on the value of the parameter is obtained in the fluid limit $T_{p}^{(0)} \rightarrow 0$ where our result is consistent with that of Goldstein (1978). Like in Inhester's simulation, we observe broader ranges of unstable modes and smaller growth rates than in the fluid description. The regime of equal equilibrium temperatures $T_{p}^{(0)}=T_{e}^{(0)}$ is illustrated in Fig. 2 which displays for $\beta=0.6$ the time evolution of the most unstable density mode $k=1.5 k_{0}$ where $k_{0}$ is the pump wavenumber, taken equal to $10 \times 2 \pi / D$ where the extension $D$ of the computational box is equal to $D=6.25 \times 2 \pi$ (in units of $L_{0}=v_{A} /\left(R_{p} \Omega_{p}\right)$ ) in the present simulations. We observe a decay instability and at later times its saturation, probably due to Landau damping rather than to mode coupling since no other mode was seen to grow during the phase where the density mode decays. Figure 3 shows in the same conditions as in Fig. 2, the variation of the parallel and perpendicular temperatures of the protons and the electrons. We note that the parallel heating of the ions is significant and that of the electrons also non-negligible (temperature growths of about $75 \%$ and $8 \%$, respectively). This electron heating supports the above statement that saturation results from Landau damping.
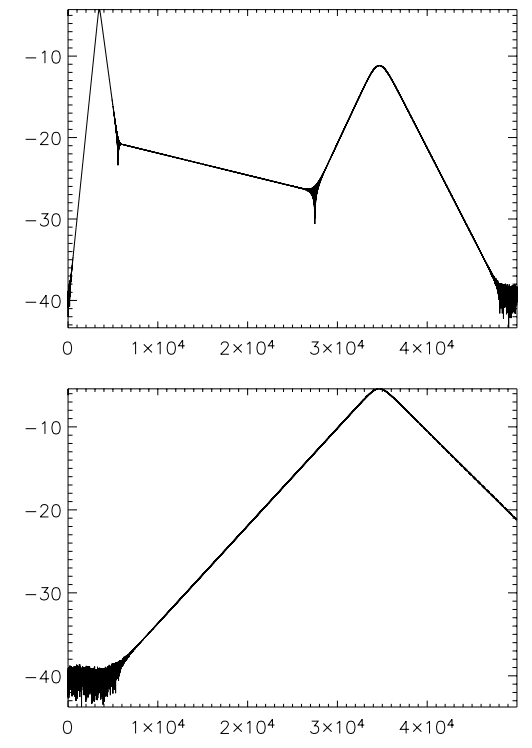

Fig. 4. Time evolution of the amplitude of the density modes $m=6$ (top) and $m=3$ (bottom) in lin-log scales, for a right-hand polarized Alfvén wave of amplitude $b_{0}=0.1, k_{0}=0.64$, in a plasma with $R_{p}=1, \beta=0.42$ and equal electron and ion equilibrium temperatures.

\section{Decay instability of dispersive Alfvén waves}

In order to consider a regime that involves dispersion but where $\beta$ is small enough for the fluid theory to qualitatively predict the linear dynamics, we assume $R_{p}=1$, $T_{p}^{(0)}=T_{e}^{(0)}, \beta_{\| p}=0.21$ (corresponding to $\beta=0.42$ ), and a forward-propagating, right-hand polarized pump with amplitude $u_{0}=0.1$ and wave number $k_{0}=4 \times 2 \pi / D=0.64$ when $D=6.25 \times 2 \pi$. Decay instability makes the density mode $m=k \times 2 \pi / D=6$ to be the most unstable at short time as seen in Fig. 4 (top). Saturation again originates from Landau damping. After a while, the mode $m=3$ starts growing (Fig. 4, bottom), which induces a second increase of the mode $m=6$ as an harmonics of $m=3$. The further dynamics corresponds to an inverse cascade involving the successive amplification of the $(m=2)$ backward and $(m=1)$ forward propagating Alfvén modes.

The regime of moderate or large $\beta$ and large wave amplitude was investigated by Vasquez (1995) using a hybrid method where electrons are treated as an isothermal fluid. A detailed analysis of the sensitivity of the decay instability growth rate to the equilibrium electron to proton temperature ratios is presented for a forward-propagating right-hand polarized Alfvén wave of amplitude $b_{0}=0.5$ and wavenumber 0.408 (normalized with the ion inertial length) propagating in a plasma with $\beta=0.45$. A periodic domain, including 8 pump wavelengths is considered. Taking $R_{p}=1$, we write the pump wavenumber $k_{0}=m_{0} \times 2 \pi / D$ with $m_{0}=8$ and $D=8 \times 2 \pi / 0.408=19.61 \times 2 \pi$ and characterize the various modes $k=m \times 2 \pi / D$ by their index $m$. For different values of the equilibrium electron to proton temperature ratio 
Table 1. Decay instability of a dispersive Alfvén wave (see text for the parameters of the runs).

\begin{tabular}{rcc}
\hline$T_{e}^{(0)} / T_{p}^{(0)}$ & $\begin{array}{c}\text { Index m of the } \\
\text { most unstable mode }\end{array}$ & $\begin{array}{c}\text { corresponding growth } \\
\text { rate in units of } \Omega_{p}\end{array}$ \\
\hline 44. & 13 & 0.087 \\
2.75 & 12 & 0.069 \\
1. & 12 & 0.059 \\
0.36 & 13 & 0.056 \\
0. & 13 & 0.056 \\
\hline
\end{tabular}

$T_{e}^{(0)} / T_{p}^{(0)}$, Table 1 provides the characteristics of the density mode with the largest grow rate measured during the linear phase. One observes that this rate decreases with $T_{e}^{(0)} / T_{p}^{(0)}$.

In the case where the pressure is constrained to obey a polytropic law of the form $\rho^{5 / 3}$, we obtain a growth rate equal to 0.092, in agreement with Goldstein (1978). Differently in the limit of hot ions, the growth rate saturates to a value roughly $40 \%$ lower than predicted by the fluid approach, whereas Vasquez (1995) hybrid simulations leads to a reduction of $57 \%$.

When $T_{e}^{(0)} / T_{p}^{(0)}=44$, the dynamics is close to a fluid regime and leads to the generation of many harmonics. A much larger numerical resolution would be required to observe the saturation of the instability.

Figure 5 displays the time variation of the ion temperatures in the run with $T_{e}^{(0)}=0$, as obtained from Landau-fluid simulations. A qualitative agreement is obtained with Vasquez (1995). In particular electrons remain cold, which justifies their description as an isothermal fluid in the hybrid code. As in the previous run, we observe an inverse cascade where the excitation is transfered to larger and larger scales (up to $m=1$ ), while the direction of the wave propagation switches at each step of the cascade, with a simultaneous increase of the ion temperature. Near $t=12000$, when the dominant Alfvén mode is $m=2$, we observe that the ion parallel temperature decreases. This event is associated with the onset of a transient instability characterized by the growth of the density mode $m=17$ and magnetic modes $m=15$ and $m=19$. The origin of this instability is unclear.

Still considering a right-handed wave, we now investigate whether decay instability can persist at relatively high values of $\beta$. Taking $R_{p}=0.1$, we show in Fig. 6 the spectrum of $b_{+}=b_{y}+i b_{z}$ for $b_{0}=0.5$ and $\beta=5$, during the linear (top panel) and the late (bottom panel) evolution phases. A decay instability is clearly visible at early time, that leads to the dominance of the (backward) $m=4$ mode, whereas the fluid theory predicts a modulational instability. In physical space, the solution evolves towards quasi-linear oscillations, associated with a rapidly decaying spectrum displaying well separated harmonics. In order to compare with the results of Vasquez (1995), we performed a simulation with the same parameters as above, except that the parameter $R_{p}$ was changed to $R_{p}=1$. In this regime, a range

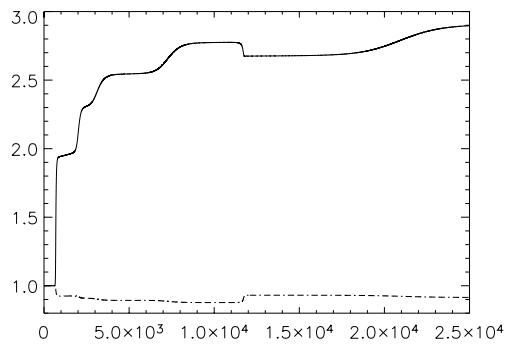

Fig. 5. Time evolution of the ion mean parallel (solid line) and perpendicular (dashed-dotted line) temperatures for a right-handed Alfvén wave with amplitude $b_{0}=0.5, k_{0}=0.408$, in a plasma with $\beta=.45, R_{p}=1$, and zero electron equilibrium temperature.
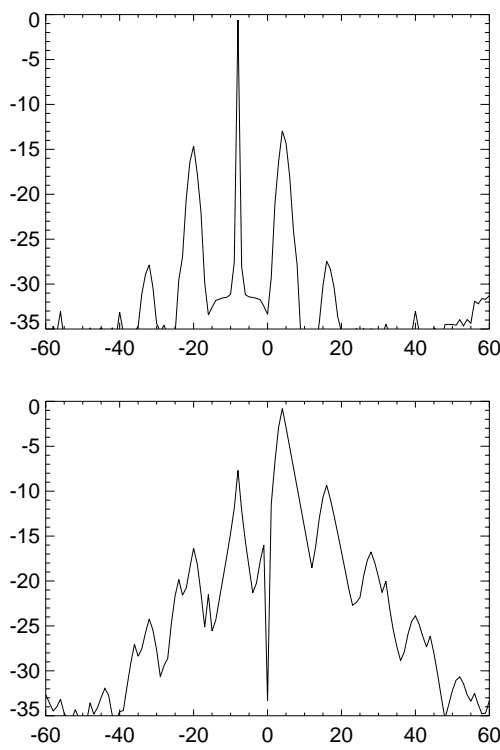

Fig. 6. Spectral density (versus the wavenumber index) in lin-log 10 scale for the (complex) quantity $b_{+}=b_{x}+i b_{y}$ (the wavenumber sign indicating a helicity and thus, after the polarization is specified, the propagation direction) at time $t=2000$ (top) and $t=3700$ (bottom) belonging, respectively, to the linear and nonlinear phases for the instability of a right-hand polarized wave with amplitude $b_{0}=0.5$, $k_{0}=0.408$ in a plasma with $\beta=5, R_{p}=0.1$ and $T_{i}^{(0)} / T_{e}^{(0)}=1.5$.

of unstable wavenumbers around $m=40$ is observed. This instability is not a numerical artifact but is rather an indication that the present regime falls outside the domain of validity of our model, since for $\beta=5$ the ion Larmor radius $\left(v_{t h} / \Omega_{p} \simeq \sqrt{\beta} v_{A} / \Omega_{p}\right)$ is comparable to the pump wavelength. The decay instability is in this case rapidly overtaken by a modulational instability leading to a turbulent behavior. Both the small-scale instability and the modulational one disappear when FLR corrections are not included in the simulation, indicating that the first-order FLR contribution retained in the present model are insufficient in a regime where the pump wavelength approaches the ion Larmor radius. A more refined model suitable to address this regime is currently under investigation. 

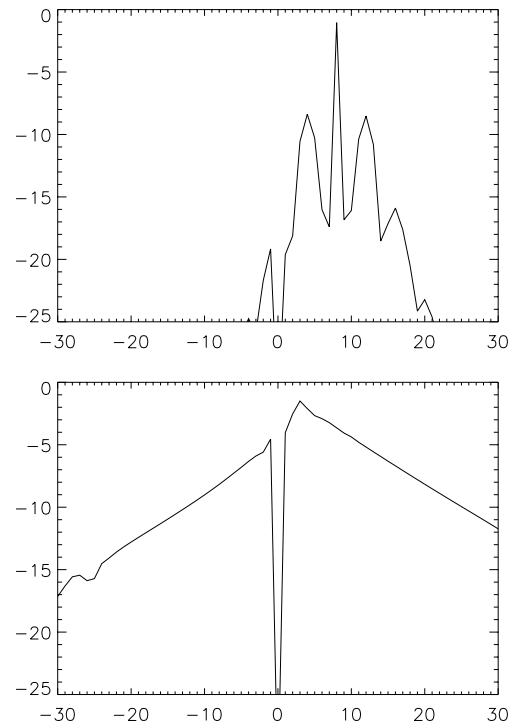

Fig. 7. Spectral density (versus the wavenumber index) in lin-log10 scale for the transverse magnetic field $b_{+}=b_{x}+i b_{y}$ at time $t=2000$ (top) and $t=3700$ (bottom) belonging, respectively, to the linear and nonlinear phases for the instability of a left-hand polarized Alfvén wave with amplitude $b_{0}=0.3, k_{0}=0.408$, in a plasma where $\beta=1.5$, $R_{p}=1$ and $T_{e}^{(0)}=2 T_{p}^{(0)}$.
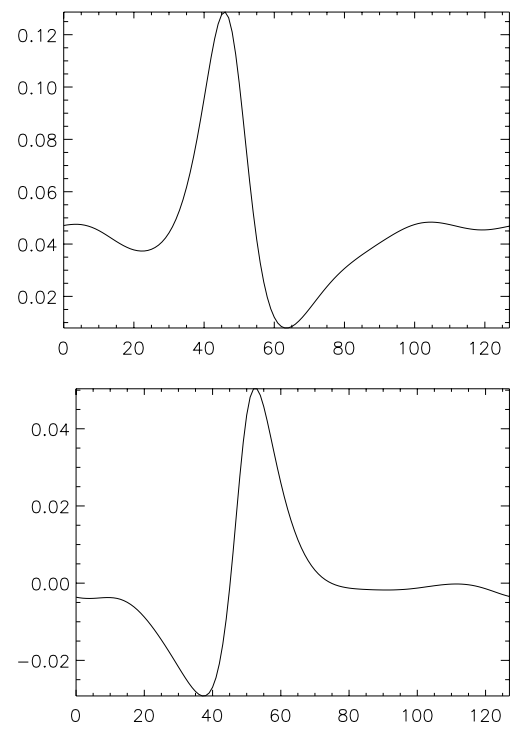

Fig. 8. Profile of $\left|b_{+}\right|^{2}$ (top) and ( $\left.\rho-1\right)$ (bottom) at $t=3700$ in the conditions of Fig. 7. The labels on the abscissa axis refer to the collocation point indices.

\section{Modulational instability}

A modulational instability of the pump is obtained in the conditions of Figs. 1 and 3 of Vasquez (1995). It corresponds to a (forward-propagating) left-hand polarized pump of amplitude $b_{0}=0.3$ and wavenumber $k_{0}=0.408=8 \times 2 \pi / D$ in a
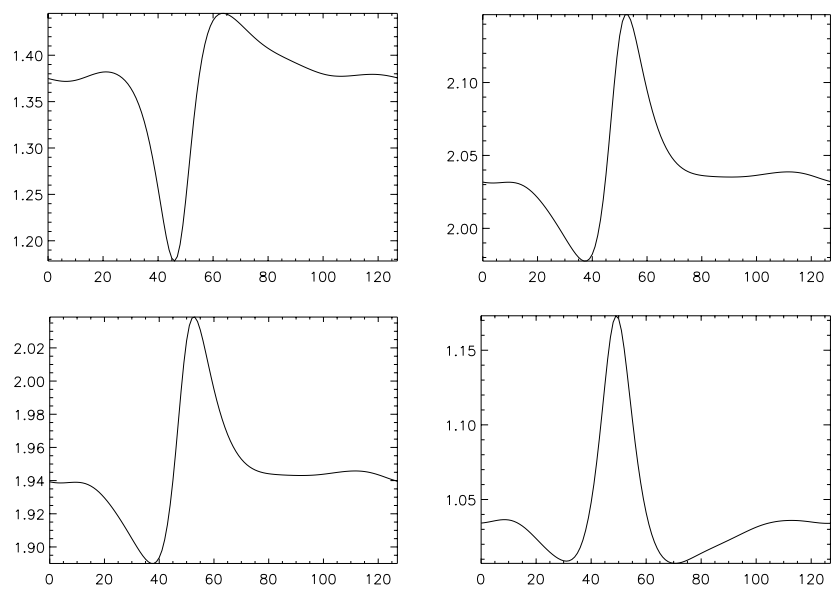

Fig. 9. Parallel (left) and transverse (right) pressures of the ions (top) and the electrons (bottom) at $t=3700$, in the conditions of Fig. 7. The labels on the abscissa axis refer to the collocation point indices.
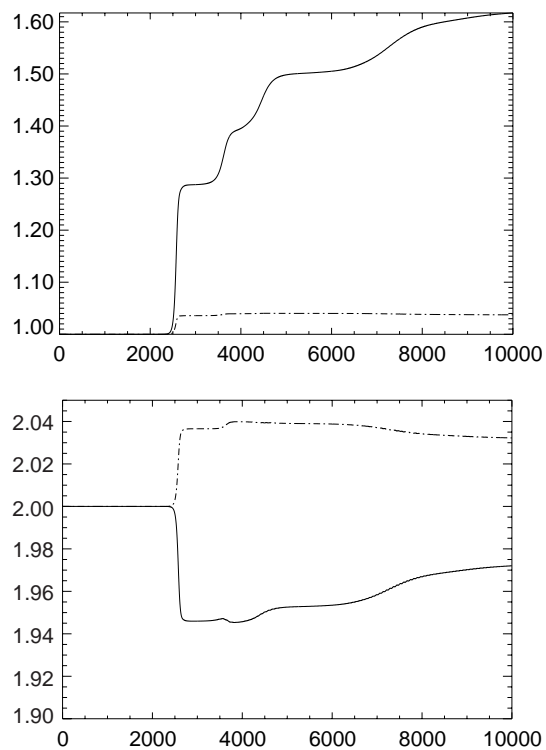

Fig. 10. Time evolution of parallel (solid lines) and transverse (dashed-dotted lines) of the ion (top) and electron (bottom) mean temperatures in the conditions of Fig. 7.

plasma with $\beta=1.5, R_{p}=1$, and $T_{i}^{(0)} / T_{e}^{(0)}=0.5$. The modulational nature of the instability is seen on Fig. 7 (top). We observe the development of modes $m=4$ and $m=12$ (clearly visible by $t=2000$ ) with similar growth rates (approximatively equal to 0.014 ). However, by $t=2600$ the former mode dominates and by $t=3700$ the mode $m=3$ emerges, while an exponential spectrum develops at small scale (Fig. 7, bottom). By $t=4700$, the mode $m=2$ is dominant. A $t=7700$, the mode $m=1$ is significantly excited and, in physical space, the Alfvén wave displays a nonlinear structure occupying all the computational box. The amplification 

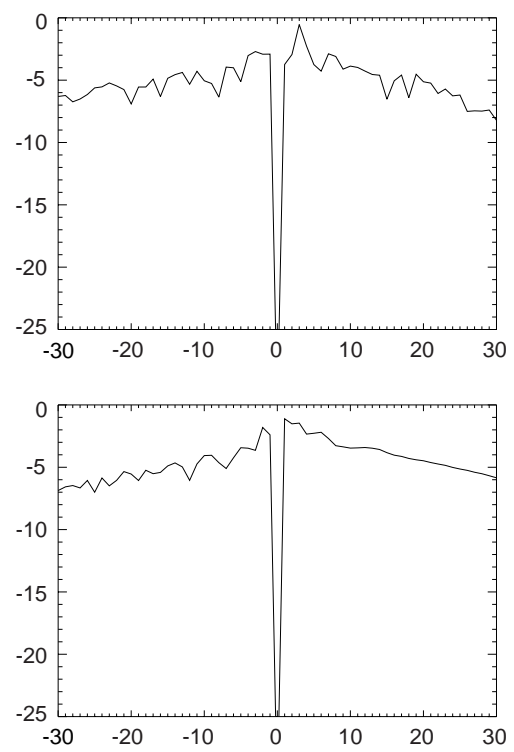

Fig. 11. Spectrum of the transverse magnetic field $b_{+}$at times $t=3100$ (top) and $t=3700$ (bottom) for an initially left-hand polarized Alfvén wave with amplitude $b_{0}=0.8$ and the same plasma parameters as in Fig. 7.
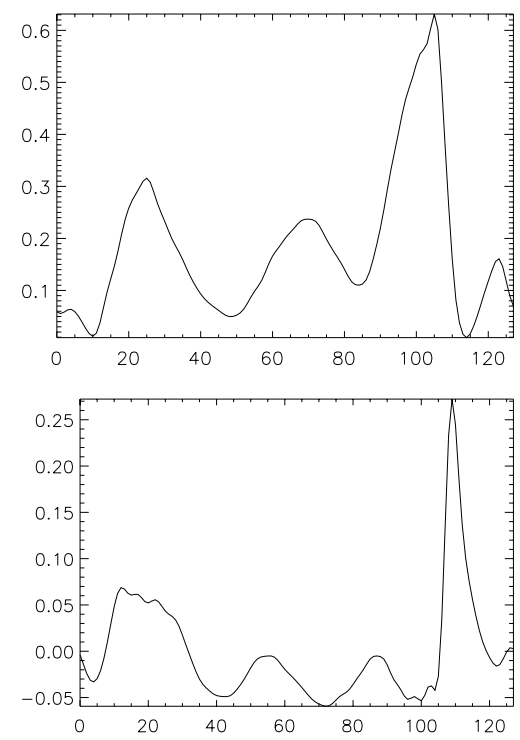

Fig. 12. Profile of $\left|b_{+}\right|^{2}$ (top) and of $(\rho-1)$ (bottom) at $t=3700$ in the conditions of Fig. 11. The labels on the abscissa axis refer to the collocation point indices.

of the lower side mode $m=4$ confirms Mjølhus and Wyller (1988) prediction based on the kinetic derivative nonlinear Schrödinger equation (Rogister, 1971) for weakly nonlinear long Alfvén waves, that a small-amplitude left-hand polarized wave is modulationally unstable for all $\beta$ (Spangler, 1989,1990; Medvedev and Diamond, 1996; Araneda, 1998). This contrasts with the fluid description that for $\beta>1.4$ predicts a dominant beat instability associated with a strong am-
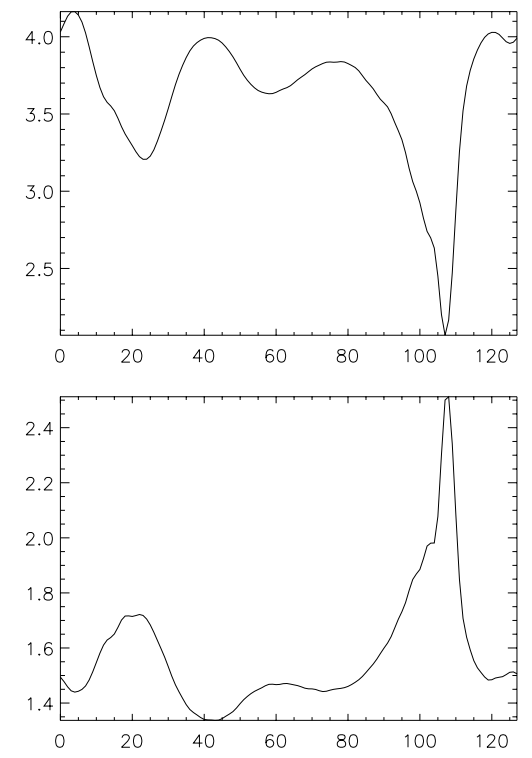

Fig. 13. Parallel (top) and transverse (bottom) ion temperatures in the conditions of Fig. 11, at time $t=3700$. The labels on the abscissa axis refer to the collocation point indices.
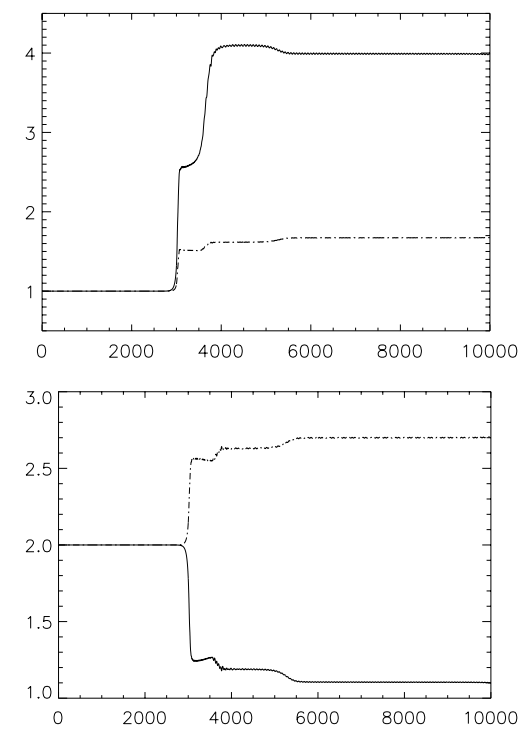

Fig. 14. Time evolution of parallel (solid lines) and transverse (dashed-dotted lines) mean temperatures of the ions (top) and the electrons (bottom) the conditions of Fig. 11.

plification of the upper Alfvén side mode. Figure 8 displays the transverse magnetic field intensity $\left|b_{+}\right|^{2}$ and the density fluctuations $\rho-1$ at time $t=3700$. Note the anti-correlation between these two quantities. Figure 9 shows the various pressures at the same time. We note that the parallel and transverse electron pressures are proportional to the density fluctuations, which justifies the description of the electrons as an isothermal fluid. Figure 10 displays the time evolu- 
tion of the mean parallel and perpendicular temperatures of the ions and the electrons for the same simulation. Note the presence of plateaux whose durations are limited by the onset of new dominant modes and the decrease of electron parallel temperature. This is a significant difference with the case where the Alfvén wave is subject to a decay instability for which the parallel electron temperature increases (possibly very slightly).

When a similar simulation is performed with a wave amplitude $b_{0}=0.8$, we still observe a dominant modulational instability (with a lower growth rate 0.011 ) as predicted by the fluid description, while Vasquez (1995) observes a dominant decay instability in this regime. The nonlinear development of this instability involves an inverse cascade and displays a turbulent regime, as exemplified in Fig. 11 that shows the spectral density of $b_{+}$at two different times. The profile of $\left|b_{+}\right|^{2}$ and of $(\rho-1)$ are shown in Fig. 12 at times $t=3700$ (where the dominant mode is $m=1$ ). Figure 13 displays at this time the parallel (top panel) and transverse (bottom panel) ion temperature profiles. Figures 14 show the time evolution of the mean ion and electron temperatures in this run. Like in Fig. 10, we observe a perpendicular heating of the electrons, an effect that is here more important due to the larger pump amplitude. Two reasons may be suggested to interpret the difference between the present simulation and the hybrid one. On the one hand, the assumption of isothermal electrons contrasts with the significant electron heating we observe with the Landau fluid. On the other hand, our simulations, in principle restricted to the weakly nonlinear regime, possibly overestimate FLR corrections in the present situation. Further developments are necessary to improve the understanding of this large amplitude regime. Note however that we observe a weak decay instability for $b_{0}=0.9$, while the modulational instability is still dominant, at least at short time (the leading modes are $m=2$ and $m=12$, the former developing at a faster rate approximatively equal to 0.0085). Note that the instability growth rate decreases as the wave amplitude is increased, as expected for a modulational instability.

\section{Conclusions}

We have used Landau-fluid simulations to analyze the instability of circularly polarized Alfvén waves propagating parallel to an ambient field in a plasma characterized by different values of the parameter $\beta$ and various ratios of the (isotropic) equilibrium temperatures of the ions and electrons. This description retains Landau damping for both ions and electrons at a linear level but ignores particle trapping. Depending on the situation, the instability is of modulational or decay type, but always results in a dominant ion heating in the parallel direction. Nevertheless, the two instabilities have different signatures on the mean perpendicular ion temperature and on both electron temperatures: for a decay-type instability ions and electrons display perpendicular cooling, while for modulational instability they are heated in the perpendicular direction. Furthermore, when Alfvén wave decay instability is saturated by Landau damping, as in the case where $T_{e}^{(0)} / T_{p}^{(0)}$ is not too large (typically smaller than a few units), the kinetic energy of the generated magnetosonic wave heats the electrons, an effect that is not retained by hybrid simulations where electrons are viewed as an isothermal fluid. The smaller-wavenumber backwardpropagating Alfvén wave that is created by the decay instability is essentially not damped and contributes to produce an inverse cascade. This evolution contrasts with the fluid regime $T_{e}^{(0)} \gg T_{p}^{(0)}$ where the onset of pump harmonics plays a dominant role in the saturation of the instability through the development of a broad spectrum at small scales. The simulation of such regimes requires relatively long integration times. Fully kinetic approach can thus hardly address these questions, while Landau-fluid models are very efficient. An interesting issue concerns the development of the inverse cascade when the computational domain is large enough to make finite size effects irrelevant, and the possible transition to a turbulent regime that can develop in this case.

\section{Appendix}

The dispersive Landau-fluid model discussed in Passot and Sulem (2004b) in the case of an electron-proton magnetized plasma with a homogeneous equilibrium state characterized by bi-Maxwellian distribution functions, reads (bold faced symbols referring to tensors)

$$
\begin{aligned}
& \partial_{t} \rho+\nabla \cdot(u \rho)=0 \\
& \partial_{t}(\rho u)+\nabla \cdot(\rho u \otimes u)+\nabla \cdot \mathbf{p}-\frac{1}{c} j \times b=0 \\
& \partial_{t} b-\nabla \times(u \times b)=-\frac{c m_{p}}{q} \nabla \times\left(\frac{1}{4 \pi \rho}(\nabla \times b) \times b-\frac{1}{\rho} \nabla \cdot \mathbf{p}_{e}\right) \\
& \partial_{t} p_{\perp r}+\nabla \cdot\left(u p_{\perp r}\right)+p_{\perp r} \nabla \cdot u-p_{\perp r} \widehat{b} \cdot \nabla u \cdot \widehat{b} \\
& +\frac{1}{2}\left(\operatorname{tr} \nabla \cdot \mathbf{q}_{r}-\widehat{b} \cdot\left(\nabla \cdot \mathbf{q}_{r}\right) \cdot \widehat{b}\right)=0 \\
& \partial_{t} p_{\| r}+\nabla \cdot\left(u p_{\| r}\right)+2 p_{\| r} \widehat{b} \cdot \nabla u \cdot \widehat{b}+\widehat{b} \cdot\left(\nabla \cdot \mathbf{q}_{r}\right) \cdot \widehat{b}=0,
\end{aligned}
$$

where $\mathbf{p}=\sum_{r} \mathbf{p}_{r}$ and $\mathbf{q}_{r}$ hold for the pressure and heat flux tensors, with $\mathbf{p}_{r}=p_{\perp r}(\mathbf{I}-\hat{b} \otimes \hat{b})+p_{\| r} \hat{b} \otimes \hat{b}+\pi_{r}$. It is convenient in Eqs. (A4) and (A5), to separate the contributions originating from the gyrotropic and non-gyrotropic heat fluxes, by writing $\mathbf{q}_{r}=\mathbf{q}_{r}^{G}+\mathbf{q}_{r}^{N G}$ with

$q_{r i j k}^{G}=q_{\| r} \widehat{b}_{i} \widehat{b}_{j} \widehat{b}_{k}+q_{\perp r}\left(\delta_{i j} \widehat{b}_{k}+\delta_{i k} \widehat{b}_{j}+\delta_{j k} \widehat{b}_{i}-3 \widehat{b}_{i} \widehat{b}_{j} \widehat{b}_{k}\right)$.

The equations for the gyrotropic pressure components involve

$\widehat{b} \cdot\left(\nabla \cdot \mathbf{q}_{r}^{G}\right) \cdot \widehat{b}=\nabla \cdot\left(\widehat{b} q_{\| r}\right)-2 q_{\perp r} \nabla \cdot \widehat{b}$ 
$\frac{1}{2}\left(\operatorname{tr}\left(\nabla \cdot \mathbf{q}_{r}^{G}\right)-\widehat{b} \cdot\left(\nabla \cdot \mathbf{q}_{r}^{G}\right) \cdot \widehat{b}\right)=\nabla \cdot\left(\widehat{b} q_{\perp r}\right)+q_{\perp r} \nabla \cdot \widehat{b}$

together with the contribution of the non-gyrotropic heat fluxes to the gyrotropic part of $\nabla \cdot \mathbf{q}_{r}$ that we denote $\left(\nabla \cdot \mathbf{q}_{r}^{N G}\right)^{G}$. In terms of diamagnetic drifts of each particle species $u_{d, r}=\frac{c}{n q|b|^{2}} b \times \nabla \cdot \mathbf{p}_{r}$ and of the current $j=\frac{c}{4 \pi} \nabla \times b$, we infer a closure approximation in the form

$$
\begin{aligned}
\left(\nabla \cdot \mathbf{q}_{e}^{N G}\right)^{G} & =2 \nabla_{\perp} \cdot\left[p_{\perp e}\left(u_{d, e}-\frac{j}{q n}\right)\right](I-\widehat{b} \otimes \widehat{b}) \\
& +\nabla_{\perp} \cdot\left[p_{\| e}\left(u_{d, e}-\frac{j}{q n}\right)\right] \widehat{b} \otimes \widehat{b}
\end{aligned}
$$

$\left(\nabla \cdot \mathbf{q}_{p}^{N G}\right)^{G}=2 \nabla_{\perp} \cdot\left[p_{\| p} u_{d, p}\right] \widehat{b} \otimes \widehat{b}$.

For the gyrotropic heat fluxes, we define

$$
\frac{q_{\| r}^{\prime}}{v_{t h, r} p_{\| r}^{(0)}}=\frac{q_{\| r}}{v_{t h, r} p_{\| r}^{(0)}}-3\left(\frac{v_{\Delta e}^{2}+v_{A}^{2}}{v_{A}^{2}}\right)\left(\frac{\Omega_{p}}{\Omega_{r}}-1\right) \frac{j_{\|}}{n q v_{t h, r}},
$$

$$
\begin{aligned}
\frac{q_{\perp r}^{\prime}}{v_{t h, r} p_{\perp r}^{(0)}} & =\frac{q_{\perp r}}{v_{t h, r} p_{\perp r}^{(0)}}+\left[\left(1+\frac{v_{\Delta e}^{2}+v_{\Delta p}^{2}}{v_{A}^{2}}\right)\left(\frac{\Omega_{p}}{\Omega_{r}}+1\right)\right. \\
& \left.-\frac{v_{\Delta p}^{2}}{v_{A}^{2}}+\frac{2 v_{\Delta r}^{2}-3 v_{t h, r}^{2}}{v_{A}^{2}} \frac{\Omega_{p}}{\Omega_{r}}\right] \frac{j_{\|}}{n q v_{t h, r}}
\end{aligned}
$$

that obey

$$
\left(\frac{d}{d t}+\frac{v_{t h, r}}{\sqrt{\frac{8}{\pi}}\left(1-\frac{3 \pi}{8}\right)} \mathcal{H} \nabla_{\|}\right) \frac{q_{\| r}^{\prime}}{v_{t h, r} p_{\| r}^{(0)}}=\frac{1}{1-\frac{3 \pi}{8}} v_{t h, r} \nabla_{\|} \frac{T_{\| r}}{T_{\| r}^{(0)}}
$$

$$
\begin{aligned}
& \left(\frac{d}{d t}-\sqrt{\frac{\pi}{2}} v_{t h, r} \mathcal{H} \nabla_{\|}\right) \frac{q_{\perp r}^{\prime}}{v_{t h, r} p_{\perp r}^{(0)}}=v_{t h, r} \nabla_{\|}\left(\left(1-\frac{T_{\perp r}^{(0)}}{T_{\| r}^{(0)}}\right) \frac{|b|}{B_{0}}\right. \\
& \left.-\frac{T_{\perp r}}{T_{\perp r}^{(0)}}+3 \sqrt{\frac{\pi}{2}} \frac{v_{t h, r}^{2}}{v_{A}^{2}} \frac{\Omega_{p}}{\Omega_{r}} \mathcal{H} \frac{j_{\|}}{n q v_{t h, r}}\right),
\end{aligned}
$$

where $v_{A}^{2}=\frac{B_{0}^{2}}{4 \pi \rho^{(0)}}$ and $v_{\Delta r}^{2}=\frac{p_{\perp r}^{(0)}-p_{\| r}^{(0)}}{\rho^{(0)}}$.

Furthermore, using the solvability conditions provided by the equations for the gyrotropic pressures, one has

$\boldsymbol{\pi}_{r} \times \widehat{b}-\widehat{b} \times \boldsymbol{\pi}_{r}=\overline{\mathbf{k}}_{r}$,

where the overline denotes the projection on the subspace complementary to the subspace spanned by the tensors $(\mathbf{I}-\widehat{b} \otimes \widehat{b})$ and $\widehat{b} \otimes \widehat{b}$, and where $\mathbf{k}_{r}$ can be decomposed into the sum of a contribution

$$
\boldsymbol{\kappa}_{r}=\frac{1}{\Omega_{r}} \frac{B_{0}}{|b|}\left[\frac{d \mathbf{p}_{r}^{G}}{d t}+(\nabla \cdot u) \mathbf{p}_{r}^{G}+\nabla \cdot \mathbf{q}_{r}+\left(\mathbf{p}_{r}^{G} \cdot \nabla u\right)^{\mathcal{S}}\right](
$$

involving the gyrotropic pressures and the heat fluxes, and of a term linear in $\boldsymbol{\pi}_{r}$

$L\left(\boldsymbol{\pi}_{r}\right)=\frac{1}{\Omega_{r}} \frac{B_{0}}{|b|}\left[\frac{d \pi_{r}}{d t}+(\nabla \cdot u) \pi_{r}+\left(\pi_{r} \cdot \nabla u\right)^{\mathcal{S}}\right]$.

It is then convenient to split the non-gyrotropic pressure as $\boldsymbol{\pi}_{r}=\boldsymbol{\pi}_{r, 1}+\boldsymbol{\pi}_{r, 2}$ with

$\pi_{r, 1} \times \widehat{b}-\widehat{b} \times \pi_{r, 1}=\overline{\kappa_{r}}$

$\boldsymbol{\pi}_{r, 2} \times \widehat{b}-\widehat{b} \times \boldsymbol{\pi}_{r, 2}=\overline{L\left(\boldsymbol{\pi}_{r, 1}\right)}+\overline{L\left(\boldsymbol{\pi}_{r, 2}\right)}$.

In a weakly nonlinear regime, the quantity $L\left(\pi_{r}\right)$ is of higher order than $\boldsymbol{\pi}_{r}$, which enables one to neglect $L\left(\boldsymbol{\pi}_{r, 2}\right)$ in Eq. (A19).

In some situations, the contribution $\pi_{r, 1}$ is sufficient and can even be simplified by approximating $\widehat{b}$ by the unit vector $\widehat{z}$ along the ambient magnetic field. Neglecting the contribution of the heat flux divergence, this leads to define $\pi_{r}^{[1]}$ by the usual gyro-viscous tensor

$\pi_{p x x}^{[1]}=-\pi_{p y y}^{[1]}=-\frac{p_{\perp p}}{2 \Omega_{p}}\left(\partial_{y} u_{x}+\partial_{x} u_{y}\right)$

$\pi_{p z z}^{[1]}=0$

$\pi_{p x y}^{[1]}=\pi_{p y x}^{[1]}=-\frac{p_{\perp p}}{2 \Omega_{p}}\left(\partial_{y} u_{y}-\partial_{x} u_{x}\right)$

$\pi_{p y z}^{[1]}=\pi_{p z y}^{[1]}=\frac{1}{\Omega_{p}}\left[2 p_{\| p} \partial_{z} u_{x}+p_{\perp p}\left(\partial_{x} u_{z}-\partial_{z} u_{x}\right)\right](\mathrm{A} 23)$

$\pi_{p x z}^{[1]}=\pi_{p z x}^{[1]}=-\frac{1}{\Omega_{p}}\left[2 p_{\| p} \partial_{z} u_{y}+p_{\perp p}\left(\partial_{y} u_{z}-\partial_{z} u_{y}\right)\right]$,

here given for the protons, the electron contribution being negligible due to the large mass ratio.

The next correction $\pi_{p}^{[2]}$ originates from terms neglected in Eq. (A18), together with the dominant contributions in Eq. (A19). It is estimated by Passot and Sulem (2004b) and appears to be usually dominated by $\partial_{t} \pi_{p}^{[1]}$.

Acknowledgements. This work was partially supported by CNRS programs "Soleil-Terre" and "Physique et Chimie du Milieu Interstellaire" and by INTAS contract 00-292.

Edited by: J. Büchner

Reviewed by: two referees

\section{References}

Abraham-Shrauner, B. and Feldman, W. C.: Nonlinear Alfvén waves in high-speed solar wind streams, J. Geophys. Res., 82, 618-624, 1877.

Araneda, A.: Parametric instabilities of parallel-propagating Alfvén waves: kinetic effects in the MHD-model, Phys. Scripta, T75, 164-168, 1998. 
Bugnon, G., Goswami, R., Passot, T., and Sulem, P. L.: Towards fluid simulations of dispersive MHD waves in a warm collisionless plasma, Proceedings of COSPAR Workshop Dynamical Processes in Critical Regions of the Heliosphere, (Ein Bokek, Israel, 3-10 March 2004) edited by: Von Steiger, R. and Gedalin, M., Adv. Space Res., Elsevier, in press, 2004.

Grad, H.: Microscopic and macroscopic models in plasma physics, in: Proceedings of the Symposium on Electromagnetics and Fluid Dynamics of Gaseous Plasmas, 37-64, Polytechnic Press of the Polytechnic Institute of Brooklyn, N.Y., 1961.

Goldstein, M. L.: An instability of finite amplitude circularly polarized Alfvén waves, Astrophys. J., 219, 700-714, 1978.

Inhester, B.: A drift-kinetic treatment of the parametric decay of large-amplitude Alfvén waves, J. Geophys. Res., 95, $10525-$ $10539,1990$.

Kulsrud, R. M.: MHD description of plasma, in: Handbook of Plasma Physics, edited by Rosenbluth, M. N. and Sagdeev, R. Z., 1 Basic Plasma Physics, edited by Galeev, A. A. and Sudan, R. N., 115-145, North Holland, 1983.

Mikkhailovskiı̌, A. B. and Smolyakov, A. I.: Theory of lowfrequency magnetosonic solitons, Sov. Phys. JETP, 61, 109-117, 1985.

Mjølhus, E. and Wyller, J.: Nonlinear Alfvén waves in a finite-betas plasma, J. Plasma Phys., 40, 299-318, 1988.

Medvedev, V. M. and Diamond, P. H.: Fluid models for kinetic effects on coherent nonlinear Alfvén waves, I. Fundamental theory, Phys. Plasmas, 3, 863-873, 1996.
Rogister, A.: Parallel propagation of nonlinear low-frequency waves in high- $\beta$ plasma, Phys. Fluids, 12, 2733-2739, 1971.

Passot, T. and Sulem, P. L.: A long-wave model for Alfvén wave trains in a collisionless plasma: I. Kinetic theory, Phys. Plasmas, 10, 3887-3905, 2003a.

Passot, T. and Sulem, P. L.: A long-wave model for Alfvén wave trains in a collisionless plasma: II. A Landau-fluid approach, Phys. Plasmas, 10, 3906-3913, 2003b.

Passot, T. and Sulem, P. L.: A fluid description for Landau damping of dispersive MHD wave, Nonlin. Proc. Geophys., 11, 245-258, 2004a,

SRef-ID: 1607-7946/npg/2004-11-245.

Passot, T. and Sulem, P. L.: A Landau-fluid model for dispersive magnetohydrodynamics, Phys. Plasmas, 11, 5173-5189, 2004b.

Snyder, P. B., Hammett, G. W., and Dorland, W.: Landau fluid models of collisionless magnetohydrodynamics, Phys. Plasmas, 4, 3974-3985, 1997.

Scalo, J. and Elmegreen, B. G.: Interstellar turbulence II: implications and effects, Ann. Rev. Astron. Astrophys., 42, 275-316, 2004.

Spangler, S. R.: Kinetic effects on Alfvén wave nonlinearity, I Ponderomotive density fluctuations, Phys. Fluids B 1, 1738-1746 1989; II The modified nonlinear wave equation, Phys. Fluids B 2, 407-418, 1990.

Vasquez, B. J.: Simulation study of the role of ion kinetics in lowfrequency wave train evolution, J. Geophys. Res., 100, 1779$1792,1995$. 